\title{
FUNCTIONS RESEMBLING QUOTIENTS OF MEASURES $\left({ }^{1}\right)$
}

\author{
BY \\ ETHAN D. BOLKER
}

Introduction. The primary concern of this paper is a generalization of the concept of expected or average value. We shall find all the set functions which resemble average values in certain ways and characterize in terms of an order structure alone those which are indeed of the form

$$
\phi(A)=\frac{1}{\mu(A)} \int_{A} f d \mu .
$$

The main theorems are stated and proved in $\S 6$. The proofs depend on Liapounoff's theorem, which says that the range of a vector valued measure is convex $(\S 1)$, on a version of dimension theory for signed measures $(\S 2)$ and on a generalization of the Radon-Nikodym theorem $(\$ 4)$.

\section{Additive sets and Liapounoff's theorem.}

1.1. Throughout this paper $\mathfrak{S}$ shall stand for a complete, atom free Boolean algebra. We shall write $1,0, \vee, \wedge,<,-$, and $\perp$ for the unit and zero of $\mathfrak{S}$, join, meet, inclusion, relative complementation and disjointness respectively. Thus $A \perp B$ means $A \leqq 1-B$. We shall write $A \subseteq$ for the principal ideal of subelements of $A$. Whenever $0 \in \mathfrak{I} \subseteq \mathfrak{S}$ we shall set $\mathfrak{I}^{\prime}=\mathfrak{I}-\{0\}$.

1.2. A nonempty subset $\mathfrak{A}$ of $\mathfrak{S}$ will be called an additive set if it is closed under the formation of proper differences and arbitrary disjoint unions:

$$
\begin{gathered}
A, B \in \mathfrak{A}, A \geqq B \Rightarrow A-B \in \mathfrak{A}, \\
\mathfrak{B} \subseteq \mathfrak{A}, B_{\alpha} \wedge B_{\beta}=\delta_{\alpha \beta} B_{\alpha} \Rightarrow \bigvee \mathfrak{B} \in \mathfrak{A} .
\end{gathered}
$$

An additive set $\mathfrak{A}$ always contains 0 . If $\mathfrak{A}$ has a greatest element that element is called the unit of $\mathfrak{A}$. If $E \in \mathfrak{A}$ then $E \mathfrak{A}=E \mathfrak{S} \cap \mathfrak{U}$ is an additive set with unit $E$. Note that $E \mathfrak{A}$ is not $\{E \wedge A \mid A \in \mathfrak{A}\} . \mathfrak{A}$ is said to be atom free if it contains a proper subelement of each of its elements.

Received by the editors October 22, 1965.

(1) Part of this research was supported by a National Science Foundation Cooperative Fellowship at Harvard University, part by NSF Grant GP 2425 at Princeton University. It is the core of the author's doctoral dissertation written under the valuable guidance of Professor Andrew M. Gleason. The construction in 7.1 which motivates the structure Theorem 6.8 is his invention. 
1.3. Lemma. If $\mathfrak{A}$ is closed under proper differences, finite disjoint unions and arbitrary increasing unions then it is additive.

Proof. Use transfinite induction to show $\mathfrak{A}$ is closed under arbitrary disjoint unions.

1.4. The additive set $\mathfrak{A}$ with unit $A$ is a measure set if there is a positive, finite, countably additive measure $\mu$ on $\mathfrak{A}$. Note that we require $\mu(A)<\infty$ and $\mu(E)=0$ only when $E=0$. A measure set $\mathfrak{A}$ can also support signed measures. A signed measure will be called strictly signed if it assumes both positive and negative values. If $v$ is a strictly signed measure on $\mathfrak{A}$ the set 3 of elements of $v$-measure zero is an additive set. All the additive sets we shall encounter are related to this simple example.

1.5. A chain is a linearly ordered set. Every chain comes equipped with its natural interval topology. Every maximal chain in an atom free additive set $\mathfrak{A}$ with unit is complete as a lattice and connected as a topological space. It follows that when $E \in \mathfrak{A}$ a maximal chain in $E \mathfrak{A}$ is maximal in $E \mathfrak{S}$. If $\mathfrak{C}$ is a maximal chain in $\mathfrak{A}^{\prime}$ then $\mathfrak{C} \cup\{0\}$ is one in $\mathfrak{A}$.

1.6. We shall say that a function on $\mathfrak{A}$ is continuous if it is continuous with respect to the interval topologies of all the chains of $\mathfrak{A}$. If $v$ is a finitely additive signed measure on $\mathfrak{A}$ then it is a measure if and only if it is continuous. It follows that maximal chains in an atom free measure set are arcs. The measure itself restricted to such a chain is a homeomorphism onto a real interval. The range of a positive measure on $\mathfrak{A}$ is thus an interval.

1.7. We can now define the principal object of our attention. Let $\Gamma$ be a chain. A nonconstant continuous function $\phi$ from $\mathcal{S}^{\prime}$ onto $\Gamma$ is an averaging function if $A \perp B$ and $\phi(A) \neq \phi(B)$ imply $\phi(A \vee B)$ is strictly between $\phi(A)$ and $\phi(B)$ in the ordering of $\Gamma$. When $A \perp B$ and $\phi(A)=\phi(B)$ we demand $\phi(A \vee B)=\phi(A)$.

1.8. Two averaging functions $\phi: \mathbb{S}^{\prime} \rightarrow \Gamma$ and $\phi^{\prime}: \mathbb{S}^{\prime} \rightarrow \Gamma^{\prime}$ are isomorphic if there is an order isomorphism $h: \Gamma \rightarrow \Gamma^{\prime}$ such that $\phi^{\prime}=h \circ \phi$. Thus the isomorphism class of $\phi$ may depend on the order structure of $\Gamma$ but does not depend on $\Gamma$ itself.

1.9. The motivating example of an averaging function comes from measure theory. If $(X, \mu)$ is a finite, atom free measure space and $v$ is a signed measure absolutely continuous with respect to $\mu$ then $\phi=v / \mu$ is an averaging function on the measure algebra $\subseteq$ of $\mu$. The phrases " $\phi=v / \mu$ " and " $\phi$ is a quotient of measures" will always refer to this situation.

1.10. Our first theorem relates averaging functions and additive sets. For each $\gamma \in \Gamma$ let $\mathfrak{Z}_{\gamma}^{\prime}=\phi^{-1}(\gamma)$, the $\gamma$-level set of $\phi$. As usual, $3_{\gamma}=3_{\gamma}^{\prime} \cup\{0\}$.

\subsection{THEOREM. $3_{\gamma}$ is an additive set.}

Proof. $3_{\gamma}$ is obviously closed under finite disjoint unions. If $A<B$ in $3_{\gamma}$ and $B-A \notin 3_{\gamma}$ then $\phi(B)$ is strictly between $\gamma=\phi(A)$ and $\phi(B-A) \neq \gamma$, contradicting the assumption that $B \in 3_{\gamma}$. The continuity of $\phi$ implies that $3_{\gamma}$ is closed 
under increasing unions so Lemma 1.3 shows $3_{\gamma}$ is an additive set. Note that for each $\left.A \in \widetilde{S}^{\prime}, A\right\}_{\phi(A)}$ is an additive set with unit $A$.

Our next task is to show that each $3_{\gamma}$ is atom free.

1.12. Lemma. Let $\mathfrak{A}$ be an atom free additive subset of $\mathfrak{S}$ with unit A. Then the range of $\phi \backslash \mathfrak{U}^{\prime}$ is a connected subset of $\Gamma$.

Proof. For each $B \in \mathfrak{A}$ choose a maximal chain $\mathfrak{C}$ in $\mathfrak{A}$ containing $B$. Then $\phi$ restricted to $\mathfrak{C}^{\prime}$ is a continuous function so $\phi\left(\mathfrak{C}^{\prime}\right)$ is a connected set $(1.5)$ containing both $\phi(B)$ and $\phi(A)$. The range of $\phi$ restricted to $\mathfrak{I}^{\prime}$ is therefore a union of connected sets which have the point $\phi(A)$ in common. Hence this range is connected.

1.13. Corollary. Lemma 1.12 applied to $\mathbb{S}$ itself shows $\Gamma$ is connected.

1.14. Theorem. If $\mathfrak{A}$ is an atom free additive subset of $\mathfrak{S}$ then $\mathfrak{A} \cap \mathfrak{Z}_{\gamma}$ is atom free.

Proof. $\mathfrak{A} \cap \mathfrak{Z}_{\gamma}$ is clearly an additive set. Suppose $0 \neq A \in \mathfrak{A} \cap \mathfrak{Z}_{\gamma}$. We must find an $A^{\prime}<A$ in $\mathfrak{A}$ such that $\phi\left(A^{\prime}\right)=\gamma$. Start by writing $A$ as a nontrivial disjoint union $B \vee D$ in $\mathfrak{A}$. This is possible since $\mathfrak{A}$ is atom free. We may assume $\phi(B) \leqq \phi(A)=\gamma \leqq \phi(D)$ since $\phi$ averages. If either equality holds we are done, so assume $\phi(B)<\gamma<\phi(D)$. Let $\mathfrak{C}$ be a maximal chain in $B \mathfrak{A}$. As $C$ increases in the connected set $\mathfrak{C}, \phi(C)$ approaches $\phi(B)$ so there is a $C \in \mathbb{C}$ with $\phi(C)<\gamma$ and $C<B$. Let $E=C \vee D<A$. Since $C \perp D, E$ is in $\mathfrak{A}$. The range of $\phi$ restricted to $E \mathfrak{U}^{\prime}$ contains $\phi(C)$, which is less than $\gamma=\phi(A)$, and $\phi(D)$, which is greater. Lemma 1.12 then implies there is an $A^{\prime}$ in $E \mathfrak{U}^{\prime}$ such that $\phi\left(A^{\prime}\right)=\gamma$.

1.15. COROLLARY. Theorem 1.14 applied to $\mathfrak{S}$ itself shows each $3_{\gamma}$ is atom free. It follows from 1.5 that maximal chains in $A \mathfrak{Z}_{\phi(A)}$ are maximal chains in $A \Im$.

1.16. Lemma. Let $\phi_{1}, \cdots, \phi_{n}$ be averaging functions on $\mathfrak{S}$. Then the level sets of the function $\phi=\left\langle\phi_{1}, \cdots, \phi_{n}\right\rangle$ with values in $\Gamma_{1} \times \cdots \times \Gamma_{n}$ are atom free additive sets.

Proof. When $\langle\gamma\rangle=\left\langle\gamma_{1}, \cdots, \gamma_{n}\right\rangle$ is in the range of $\phi$ then $\mathcal{Z}_{\langle\gamma\rangle}=3_{\gamma_{1}} \cap \cdots \cap \mathcal{Z}_{\gamma_{n}}$, which is clearly an additive set. Induction shows it is atom free.

Some corollaries of this lemma lead directly to a theorem of Liapounoff's [3]. The idea behind our proof is implicit in Halmos' [2]. However by making explicit use of additive sets we are able to rest more of the proof on elementary topological facts about chains and less on explicit measure theoretic constructions. Our proof is more elementary than Dubin and Spanier's [1], which uses the BorsukUlam theorem, but the preliminary lemma we derive below is weaker than theirs.

1.17. Lemma (Simultaneous Proportionality). Suppose $v_{1}, \cdots, v_{n}$ are signed measures on the atom free measure algebra $\mathfrak{S}$. Let $v$ be the vector valued measure 
$\left\langle v_{1}, \cdots, v_{n}\right\rangle$. For every $A$ in $\subseteq$ and real $t$ between 0 and 1 there is $a B<A$ such that $v(B)=\operatorname{tv}(A)$.

Proof. Choose some measure $\mu$ on $\mathfrak{\Im}$ which vanishes only at 0 and set $\phi=v / \mu$. Lemma 1.16 implies that the additive set $A 3_{\phi(A)}$ is atom free. Choose a maximal chain $\mathfrak{C}$ in $A 3_{\phi(A)}$. Then 1.6 shows there is a $B$ in $\mathfrak{C}$ such that $\mu(B)=t \mu(A)$. Since

$$
v(A) / \mu(A)=\phi(A)=\phi(B)=v(B) / \mu(B)
$$

it follows that $v(B)=t v(A)$.

1.18. Corollary (Simultaneous Bisection). There is $a \quad B<A$ such that $v(B)=v(A) / 2$. Note for future reference that in the construction in 1.18 both $B$ and $A-B$ have this property.

The restriction in 1.17 that $\subseteq$ be a measure algebra and the seemingly ad hoc introduction of the positive measure $\mu$ could be avoided were we willing to assume the Hahn decomposition theorem and the resulting construction of the total variation measures $\left|v_{i}\right|$. Suppose the vector valued measure $v$ has at least one atom free component. Set $\mu=\left|v_{1}\right|+\cdots+\left|v_{n}\right|$. Since $v_{i}$ is absolutely continuous with respect to $\mu$ the argument in 1.17 applies when $\subseteq$ is the measure algebra of $\mu$.

1.19. THEOREM (LIAPOUNOFF). The range of the vector valued measure $v$ on the atom free measure algebra $\mathfrak{S}$ is a convex subset of $\boldsymbol{R}^{n}$. It is also closed, but we shall not prove that.

Proof. Given $A$ and $B$ in $\subseteq$ and $x$ between 0 and 1 we must find a $C$ in $\subseteq$ such that $v(C)=x v(A)+(1-x) v(B)$. Start by applying 1.17 to $A-B$ with $t=x$ and to $B-A$ with $t=1-x$ to produce $D \leqq A-B$ and $E \leqq B-A$ satisfying

$$
v(D)=x v(A-B) \text { and } v(E)=(1-x) v(B-A) .
$$

Then set $C=D \vee(A \wedge B) \vee E$. This union is disjoint, so

$$
\begin{aligned}
v(C) & =v(D)+v(A \mathrm{I} \wedge B)+v(E) \\
& =x v(A-B)+[x+(1-x)] v(A \wedge B)+(1-x) v(B-A) \\
& =x v(A)+(1-x) v(B) .
\end{aligned}
$$

As corollaries to Liapounoff's theorem we shall derive some new, useful results on the uniqueness of signed measures.

1.20. THEOREM. Suppose $v_{1}$ and $v_{2}$ are strictly signed measures such that for $A \neq 0, v_{2}(A) \leqq 0$ implies $v_{1}(A)<0$. Then the set I of positive numbers $t$ such that $v_{2} \geqq t v_{1}$ is a closed interval.

Proof. $I$ is clearly connected. If it is not empty it contains its supremum and infimum provided these are not $\infty$ or 0 respectively. Consider the measure 
$v=\left\langle v_{1}, v_{2}\right\rangle$ with values in the plane. The hypotheses imply that the range $X$ of $v$ meets the open first and third quadrants but not the open fourth quadrant. $I$ clearly contains just those $t>0$ for which $X$ lies above the line $y=t x$. It is therefore bounded and bounded away from zero. The convex set $X$ can be separated from the fourth quadrant by such a line, so $I$ is not empty.

1.21. A convex subset $X$ of $\boldsymbol{R}^{n}$ has no interior if and only if it is contained in a hyperplane. If $X$ meets both the open half spaces into which a hyperplane $P$ separates $\boldsymbol{R}^{n}$ then $X$ has an empty interior if $X \cap P$ has an empty interior relative to $P$.

1.22. THEOREM. Suppose $\mu_{1}$ and $\mu_{2}$ are atom free signed measures such that for some $\alpha$ in the interior of the range of $\mu_{1}, \mu_{1}(A)=\mu_{1}(B)=\alpha$ implies $\mu_{2}(A)=\mu_{2}(B)$. Then $\mu_{2}=\beta \mu_{1}$ for some real $\beta$.

Proof. Let $X$ be the range of the vector measure $\left\langle\mu_{1}, \mu_{2}\right\rangle . X$ meets both open half planes determined by the line $x=\alpha$ but meets that line in a single point. $X$ is therefore contained in a line (1.21). Since $0 \in X$ that line is of the form $y=\beta x$. Then $\mu_{2}=\beta \mu_{1}$.

1.23. Corollary. A probability measure $\mu$ on $\mathfrak{S}$ is completely determined by knowledge of the elements of $\mathbb{S}$ with measure one half. This fact is implicit in Halmos' proof of Liapounoff's theorem. A strictly signed measure is determined up to a scalar factor by knowledge of the additive set of elements of measure zero.

In the next section we shall try to find constructive analogues of these passive assertions.

2. Measures from equivalence relations. In this section we shall discuss how a measure $\mu$ on an additive set can be recovered from data about the partition into classes of elements of equal measure which it defines. We have just seen that complete knowledge of any single equivalence class suffices to determine $\mu$. Nevertheless, in each of the two cases we consider we shall have to allow ourselves more information. We use the entire partition to reconstruct a positive measure. The zero equivalence class and a Hahn decomposition suffice for a strictly signed measure. The underlying principle we exploit for positive measures is not new. The idea stems historically from dimension theory; see, for example, [5]. Maharam's work [4] is closest in spirit to ours.

The particular theorem we are about to prove has hypotheses easily verifiable in the applications we wish to make. Some parts of it express new points of view on old ideas. The model for the proof is the characterization of the unit interval as the complete separable ordered local semigroup. Since the techniques are well known we shall often just outline arguments. The results of this section are independent of Liapounoff's theorem though we shall use information about chains in atom free additive sets. 
2.1. Theorem. Let $\mathfrak{A}$ be an atom free additive set with unit 1 and $\equiv$ an equivalence relation on $\mathfrak{A}$ which satisfies

I. Additivity. If $E_{1} \perp E_{2}, F_{1} \perp F_{2}, E_{1} \equiv F_{1}$ and $E_{2} \equiv F_{2}$ then $E_{1} \vee E_{2} \equiv F_{1} \vee F_{2}$.

II. Strong Comparability. Every maximal chain in $\mathfrak{A}$ contains a representative of every $\equiv$ equivalence class.

III. Comparability. If $A$ and $B$ are in $\mathfrak{A}$ then one of them is equivalent to a subelement of the other.

IV. Boundedness and Nontriviality. No $A$ is equivalent to a subelement of itself.

Then there is a unique measure $\mu$ on $\mathfrak{A}$ such that $\mu(1)=1$ and $A \equiv B$ if and only if $\mu(A)=\mu(B)$.

The conclusion of the theorem is unsurprising but the hypotheses seem weak. We do not assume $\mathfrak{A}$ is a measure set; we do not even know, a priori, that it satisfies the countable chain condition. Neither do we assume $\equiv$ is countably additive, or even continuous, except indirectly in II.

The following easy analogue of the Schroder-Bernstein theorem is critical in the proof.

2.2. LEMMA.

$$
\begin{aligned}
& A \equiv D \\
& \wedge \\
& B \equiv C \text { is impossible. }
\end{aligned}
$$

Proof. Try to apply III to $A$ and $C$. Because of the symmetry of the square it suffices to show that $A \equiv E \leqq C$ is impossible. But if there were such an $E$ then $D \equiv A \equiv E \leqq C<D$ would contradict IV.

Now let $\delta: \mathfrak{A} \rightarrow \Delta$ be the natural map of $\mathfrak{A}$ onto the set of $\equiv$ equivalence classes. Let $\delta(0)=0$ and $\delta(1)=1$. Suppose $\mathfrak{C}$ is a maximal chain in $\mathfrak{A}$. It follows from II and Lemma 2.2 that $\delta \uparrow \mathfrak{C}$ is bijective and that the order structure on $\Delta$ induced by $\delta \uparrow \mathfrak{C}$ is independent of $\mathfrak{C} . \Delta$ is therefore complete and connected in the interval topology of this order structure.

The following lemma, whose routine proof we omit, allows us to define subtraction in $\Delta$.

2.3. Lemma. $B<A, F<E, A \equiv E$ and $B \equiv F$ imply $A-B \equiv E-F$.

When $\beta<\alpha$ in $\Delta$ choose representatives $B<A$ in $\mathfrak{U}$ and set $\alpha-\beta=\delta(A-B)$. For each fixed $\alpha$ the subtraction map $\beta \rightarrow \alpha-\beta$ is a continuous function of $\beta$ in the segment of $\Delta$ below $\alpha$. If $\alpha \leqq 1-\beta$ then $\alpha$ and $\beta$ have disjoint representatives $A$ and $B$ and $\alpha+\beta$ is consistently defined as $\delta(A \vee B)$. Addition is a continuous, monotonic function of each variable. It is associative and commutative where it is defined because disjoint union enjoys these properties. 
The measure $\mu$ on $\mathfrak{A}$ which we seek must, by definition, factor through $\delta$ and map $\Delta$ isomorphically (as an ordered set with its additive structure) onto the unit interval $I$. To simplify our notation we shall construct the inverse $\sigma$ of this isomorphism. First we locate $\sigma\left(2^{-n}\right)$, then $\sigma$ for the dyadic rationals $D$ and then extend by continuity.

Let $\sigma(1)=\sigma\left(2^{0}\right)=\delta(1)=1$ in $\Delta$. Then define $\sigma\left(2^{-n}\right)$ inductively. Suppose $\sigma\left(2^{-n+1}\right)$ has been defined. Then the interval $\left[0, \sigma\left(2^{-n+1}\right)\right]$ in $\Delta$ is a connected set on which the function $\alpha \rightarrow \sigma\left(2^{-n+1}\right)-\alpha$ is monotonic, order reversing and continuous. There is therefore a unique $\sigma\left(2^{-n}\right) \in \Delta$ for which $\sigma\left(2^{-n}\right)+\sigma\left(2^{-n}\right)=\sigma\left(2^{-n+1}\right)$.

2.4. LEMMA. $\sigma\left(2^{-n}\right) \rightarrow 0$ in $\Delta$.

Proof. We know $\sigma\left(2^{-n}\right)$ is a decreasing sequence in the ordering of $\Delta$. Let $\alpha=\inf \sigma\left(2^{-n}\right)$. Suppose $\alpha>0$. Then find two disjoint members $C_{1}$ and $D_{1}$ in $\sigma(1 / 2)$. Let $\mathbb{C}$ be a maximal chain containing $C_{1}$ and $\mathfrak{D}$ a maximal chain containing $D_{1}$. Let $C_{n}=\sigma\left(2^{-n}\right) \cap \mathbb{C}, D_{n}=\sigma\left(2^{-n}\right) \cap \mathfrak{D}, C=\alpha \cap \mathbb{C}$ and $D=\alpha \cap \mathfrak{D}$. Then $0<C<C_{n}$ and $0<D<D_{n}$ for all $n$. Since $C_{1} \perp D_{1}$ we know $C_{n} \perp D_{n}$ and $C \perp D$. Thus $C \vee D<C_{n} \vee D_{n}$ for all $n$ and these are disjoint unions Hence

$$
\begin{aligned}
\delta(C \vee D)<\delta\left(C_{n} \vee D_{n}\right) & =\delta\left(C_{n}\right)+\delta\left(D_{n}\right) \\
& =\sigma\left(2^{-n}\right)+\sigma\left(2^{-}\right) \\
& =\sigma\left(2^{-n+1}\right)
\end{aligned}
$$

for all $n$. Therefore $\delta(C \vee D) \leqq \alpha$. But since $C<C \vee D, \alpha<\delta(C \vee D)$, a contradiction. $\alpha$ must be 0 in $\Delta$.

Lemma 2.4 implies $\sigma(D)$ is dense in $\Delta$. It is then easy to prove that $\sigma$ extends uniquely to a continuous local semigroup isomorphism of $I$ onto $\Delta$. Since addition is "countably additive" on $I$ the measure $\mu=\sigma^{-1} \delta$ on $\mathfrak{A}$ is countably additive and satisfies the requirements of Theorem 2.1, which has therefore been proved.

2.5. Corollary. A finitely additive probability measure on a complete, atom free Boolean algebra is countably additive if it vanishes only at 0 and if every maximal chain contains an element of measure $x$ for every $x$ between 0 and 1 .

Later we shall need the following slight strengthening of the uniqueness assertion of Theorem 2.1.

2.6. LEMMA. Let $\mu_{1}$ and $\mu_{2}$ be measures on the additive set $\mathfrak{A}$, which need not have a unit. Suppose that $\mu_{1}(A)=0$ only when $A=0$ and that $\mu_{1}(A)=\mu_{1}(B)$ if and only if $\mu_{2}(A)=\mu_{2}(B)$. Then $\mu_{1}$ is a positive multiple of $\mu_{2}$.

Proof. For each $A \in \mathfrak{A}$ apply Theorem 2.1 on $A \mathfrak{A}$. There $\mu_{1}=\lambda_{A} \mu_{2}$. The numbers $\lambda_{A}$ are independent of $A$ since given $A$ and $A^{\prime}$ both $A \mathfrak{U}$ and $A^{\prime} \mathfrak{A}$ will contain elements of $\mu_{1}$ measure $x$ when $x$ is sufficiently small. 
The rest of this section is devoted to reconstructing a strictly signed measure on all of $\mathfrak{S}$ from its Hahn decomposition and zero elements.

2.7. Theorem. Let 3 be an atom free additive subset of $\mathfrak{S}$ and $P_{0} \in \mathfrak{S}$. Set $N_{0}=1-P_{0}, \mathfrak{P}=P_{0} \mathfrak{S}$ and $\mathfrak{N}=N_{0} \mathfrak{S}$. Write $N$ and $P$ for generic members of $\mathfrak{N}$ and $\mathfrak{P}$ respectively. If $N \vee P \in \mathcal{Z}$ we shall say that $N$ balances $P$. Assume $\mathfrak{N}, 3$ and $\mathfrak{P}$ satisfy

$I^{\prime} . \mathfrak{N} \cap \mathbb{Z}=\mathfrak{P} \cap \mathbb{Z}=\{0\}$.

II'. If $N \in \mathfrak{N}$ and $P \in \mathfrak{P}$ then one balances a subelement of the other.

III'. If $N$ balances $P$ and $\mathfrak{C}(\mathfrak{D})$ is a maximal chain in $\mathfrak{P}(\mathfrak{N})$ then there is a $C \in \mathbb{C}(D \in \mathfrak{D})$ which balances $N(P)$.

Then there is a unique strictly signed measure $v$ on $\mathfrak{S}$ such that $v\left(P_{0}\right)=1$ and $v(A)=0$ if and only if $A \in 3$. It follows that $N_{0}, P_{0}$ is a Hahn decomposition for $v$.

Many of the statements in the proof outlined below have obvious analogues obtained by interchanging $\mathfrak{P}$ and $\mathfrak{N}$. We shall not always mention them or prove them.

2.8. Call a $P \in \mathfrak{P}$ small if it is balanced by some $N \in \mathfrak{N}$. The following lemma shows that subelements of small elements are small and that either all of $\mathfrak{P}$ or all of $\mathfrak{N}$ is small.

2.9. LEMMA. If $N$ balances $P$ and $Q \leqq P$ then some subelement of $N$ balances $Q$.

Proof. We may assume $Q<P$. Apply II' to $N$ and $Q$. It suffices to show that $Q^{\prime}<Q$ cannot balance $N$. But were this to occur, $N \vee P-N \vee Q^{\prime}=P-Q^{\prime} \neq 0$ would be in $\mathfrak{P} \cap \mathcal{Z}$, contradicting $I^{\prime}$.

2.10. Define two small elements of $\mathfrak{P}$ to be equivalent, written $\equiv$, if both are balanced by the same $N$ in $\mathfrak{N}$. $\equiv$ is clearly reflexive and symmetric. The next lemma shows it is transitive.

2.11. Lemma. If $N_{1}$ balances $P_{1}$ and $P_{1}{ }^{\prime} \equiv P_{2}$ then $N_{1}$ balances $P_{2}$.

Proof. Choose an $N_{2}$ which balances both $P_{1}$ and $P_{2}$. If $N_{1} \perp N_{2}$ and $P_{1} \perp P_{2}$ the result follows from the additivity of 3 and the observation

$$
\left(N_{1} \vee P_{1}\right) \vee\left(N_{2} \vee P_{2}\right)=\left(N_{2} \vee P_{1}\right) \vee\left(N_{1} \vee P_{2}\right)
$$

Now suppose $N_{1} \perp N_{2}$ but $P_{1} \wedge P_{2}=B>0$. Apply Lemma 2.9 to find elements $C_{i}<N_{i}$ such that $C_{i} \vee \vee B \in 3, i=1,2$. The original argument then applies to $N_{1}-C_{1}, N_{2}-C_{2}, P_{1}-B$ and $P_{2}-B$, allowing us to deduce that $N_{1}-C_{1}$ balances $P_{2}-B$. Since $C_{2} \vee B$ is in $3, N_{1} \vee P_{2} \in 3$.

Finally, suppose $C=N_{1} \wedge N_{2}>0$. Apply II' to $B$ and $C$; assume this produces a $D<B$ which balances $C$. Then apply the preceding argument to $P_{1}-D, P_{2}-D$, $N_{1}-C$ and $N_{2}-C$. 
2.12. We wish to show $\equiv$ satisfies the hypotheses of Theorem 2.1. Verification of II, III and IV is routine. The finite additivity is easy to check. Suppose $P_{1} \equiv Q_{1}$, $P_{2} \equiv Q_{2}, P_{1} \perp P_{2}, Q_{1} \perp Q_{2}$ and $P_{1} \vee P_{2}$ is small. Let $N$ balance $P_{1} \vee P_{2}$ and select $N_{1}<N$ balancing $P_{1}$ (2.9). Then $N_{1}$ balances $Q_{1}$ (2.11) and $N_{2}=N-N_{1}$ balances $P_{2}$ and hence $Q_{2}$. Therefore $N=N_{1} \vee N_{2}$ balances $Q_{1} \vee Q_{2}$ which is thus equivalent to $P_{1} \vee P_{2}$.

Recall that all of $\mathfrak{P}$ or $\mathfrak{N}$ is small (2.8); assume the former. Then Theorem 2.1 applies to $\equiv$ on $\mathfrak{P}$; let $v$ be the measure so constructed. Extend $v$ to the small elements of $\mathfrak{N}$ by setting $v(N)=-v(P)$ when $N$ balances $P$. It is easy to see that every $N \in \mathfrak{N}$ is a disjoint union of small elements and that the obvious extension of $v$ to all of $\mathfrak{N}$ is consistent and defines a countably additive negative measure on $\mathfrak{R}$. Were $v$ to assume the value $-\infty$ it would do so on an entire maximal chain, contradicting the fact that every maximal chain in $\mathfrak{N}^{\prime}$ contains a small element. Finally, extend $v$ to all of $\subseteq$ by setting $v(A)=v\left(A \wedge P_{0}\right)+v\left(A \wedge N_{0}\right)$. The corresponding definition with a minus sign yields the positive measure $|v|$ on $\mathfrak{S}$, which shows $\mathfrak{S}$ is a measure algebra.

3. When are $\nu / \mu$ and $\nu^{\prime} / \mu^{\prime}$ isomorphic? We begin this section with a technical corollary of the simultaneous bisection argument in 1.18 .

3.1. Lemma. Let $v$ be a vector valued signed measure. If $A_{1}, \cdots, A_{k}$ are in $\mathfrak{S}$ there are mutually disjoint elements $B_{1}, \cdots, B_{k}$ such that $B_{j}<A_{j}$ and $v\left(B_{j}\right)$ $=2^{-k} v\left(A_{j}\right)$ for $j=1, \cdots, k$.

Proof. Let $D_{1}, \cdots, D_{r}$ be the atoms of the subalgebra of $\subseteq$ generated by $A_{1}, \cdots, A_{k}$. Then the elements $D_{m}$ are pairwise disjoint in $\subseteq$ and $r \leqq 2^{k}$. Furthermore, $A_{j}=\bigvee_{D_{m} \leqq A_{j}} D_{m}$. Apply the Simultaneous Bisection Corollary $1.18 k$ times inside each $D_{m}$ to produce disjoint subelements $E_{m}^{1}, \cdots, E_{m}^{2^{k}}$ each of which satisfies $v\left(E_{m}^{i}\right)=2^{-k} v\left(D_{m}\right)$. Then set $B_{j}=\bigvee_{D_{m} \leqq A_{j}} E_{m}^{j}$. Note for later use that for each $m$ we may permute the upper indices of the elements $E_{m}^{j}$ and construct different elements $B_{j}$ which work equally well.

3.2. Lemma. Suppose $A \perp B$ and $\phi(A) \neq \phi(B)$. Let $\mathbb{C}$ be a maximal chain in $A \mathfrak{3}_{\phi(A)}$. Then the function $g$ defined by $g(C)=\phi(C \vee B)$ is a homeomorphism of $\mathfrak{C}$ onto the interval $[\phi(A \vee B), \phi(B)]$ in $\Gamma$.

Proof. $g$ is continuous since $\phi$ is. $g(0)=\phi(B)$ and $g(A)=\phi(A \vee B)$. Since the domain of $g$ is connected, $g$ will be a homeomorphism provided it is strictly monotonic. But suppose $C_{1}>C_{2}$ in $C$. Then $C_{1} \vee B=\left(C_{2} \vee B\right) \vee\left(C_{1}-C_{2}\right)$. Since $\phi(B) \neq \phi(A)=\phi\left(C_{1}\right)=\phi\left(C_{2}\right)=\phi\left(C_{1}-C_{2}\right)$ and $\phi$ averages, $\phi\left(C_{1} \vee B\right)$ is nearer $\phi(B)$ than is $\phi\left(C_{2} \vee B\right)$.

3.3. Definition. The ordered triple $\left\langle A_{1}, A_{2}, B\right\rangle$ of disjoint elements of $\mathfrak{S}$ is a test set configuration if $\phi\left(A_{1}\right)=\phi\left(A_{2}\right) \neq \phi(B)$ and $\phi\left(A_{1} \vee B\right)=\phi\left(A_{2} \vee B\right)$. 
determined modulo $\mu$ up to a change of scale. No more could be hoped for from the data.

It is easy to see why the qualitative data convey so much information. In order to compute the average value of $f$ on $E$ we must first perform the experiment corresponding to the observable given by the characteristic function of $E$.

4. The Radon-Nikodym theorem. In this section we establish a lattice theoretic version of the Radon-Nikodym theorem for an averaging function $\phi$ on a complete Boolean algebra $\mathfrak{S}$. We temporarily drop the assumption that $\mathfrak{S}$ is atom free and that $\phi: \Im^{\prime} \rightarrow \Gamma$ is onto in order to deduce the standard measure theoretic version from ours when $\phi=v / \mu$ and $\Gamma=R$.

4.1. Definition. A map $\gamma \rightarrow E_{\gamma}$ from $\Gamma$ to $\mathfrak{S}$ is a spectral family if

$$
\begin{gathered}
E_{\gamma} \text { increases with } \gamma, \\
E_{\gamma}=\bigwedge_{\beta>\gamma} E_{\beta}, \\
\vee E_{\gamma}=1 \text { and } \wedge E_{\gamma}=0 .
\end{gathered}
$$

It is clear that when $\mathfrak{S}$ is a measure algebra and $\Gamma=\boldsymbol{R}$ there is a one-to-one correspondence between measurable functions $f$ and spectral families given by setting $E_{\gamma}=f^{-1}((-\infty, \gamma])$. When $f=d v / d \mu E_{\gamma}$, is the largest element of $\mathfrak{S}$ on every subelement of which $\phi$ is less than or equal to $\gamma$. This is the clue to the generalization of the Radon-Nikodym theorem which follows.

4.2. LeMMA. $\phi \uparrow E \Im \leqq \gamma$ and $\phi \uparrow F \subseteq \leqq \gamma$ imply $\phi \uparrow(E \vee F) \subseteq \leqq \gamma$.

Proof. Suppose $G \leqq E \vee F$. Then $G=[G \wedge E] \vee[G \wedge(F-E)]$ is a disjoint union. Since $\phi$ averages, $\phi(G) \leqq \gamma$.

This lemma shows that the set $\mathfrak{U}_{\gamma}$ of members $E$ of $\mathfrak{S}$ such that $\phi \uparrow E \mathbb{S}^{\prime} \leqq \gamma$ is an ideal and not just an additive set. Unfortunately the easy proof above rests heavily on the Boolean properties of relative complementation and distributivity. This obstructs the immediate extension of the results which follow to more general orthocomplemented lattices.

4.3. Lemma. $\mathfrak{U}_{\gamma}$ is not empty. That is, if $\gamma \in \Gamma$ is not less than every member of $\Gamma_{0}=$ range $\phi$ then there is an $E$ in $\mathfrak{S}$ such that $\phi \uparrow E \Im^{\prime} \leqq \gamma$.

Proof. If $\gamma$ is the least member of $\Gamma_{0}$ then any $E$ for which $\phi(E)=\gamma$ will do. If $\gamma$ is not the least member of $\Gamma_{0}$ choose an $F$ in $\mathfrak{S}$ for which $\phi(F)<\gamma$. Let $\mathfrak{M}=\{G \leqq F \mid \phi(G) \geqq \gamma\}$. If $\mathfrak{M}$ is empty the lemma is proved by setting $E=F$. Otherwise let $G$ be the join of a maximal chain $\mathfrak{C}$ in $\mathfrak{M}$. Clearly $G$ is a maximal member of $\mathfrak{M}$ and is not equal to $F$. Set $E=F-G>0$; suppose $D \leqq E$. Then $D \perp G$ so the maximality of $G$ implies $D \vee G$ is not in $\mathfrak{M}$. Since $\phi(D \vee G)$ is 
3.4. Lemma. If $\phi=v / \mu$ and $\left\langle A_{1}, A_{2}, B\right\rangle$ is a test set configuration then

$$
\begin{aligned}
& v\left(A_{1}\right)=v\left(A_{2}\right), \\
& \mu\left(A_{1}\right)=\mu\left(A_{2}\right)
\end{aligned}
$$

and the quadruple

$$
\left(\phi\left(A_{1}\right), \phi\left(A_{1} \vee B\right) ; \phi\left(A_{1} \vee B \vee A_{2}\right), \phi(B)\right)
$$

is harmonic in $\boldsymbol{R}$.

Proof. Compute.

3.5. LEMMA. If $\phi=v / \mu$ and $\alpha<\gamma<\beta$ are in the range of $\phi$ then there is a test set configuration $\left\langle A_{1}, A_{2}, B\right\rangle$ such that $\phi\left(A_{1}\right)=\alpha, \phi(B)=\beta$ and $\phi\left(A_{1} \vee B \vee A_{2}\right)=\gamma$.

Proof. Apply Lemma 3.1 to the measure $\langle v, \mu\rangle$ to find disjoint elements $A$ and $B$ for which $\phi(A)=\alpha$ and $\phi(B)=\beta$. Then use Lemma 3.2 to replace either $A$ or $B$ by a subelement of itself to guarantee that $\phi(A \vee B)=\gamma$. Finally, apply the Simultaneous Bisection Corollary 1.18 to $\langle v, \mu\rangle$ on $A$ to produce disjoint halves $A_{1}$ and $A_{2}$ of $A$. Then $\left\langle A_{1}, A_{2}, B\right\rangle$ is the configuration we seek.

3.6. THEOREM. Let $h$ be an increasing function on the range $\Gamma$ of $\phi=v / \mu$. Then $h \circ \phi$ is a quotient of measures if and only if $h$ is the restriction to $\Gamma$ of a fractional linear transformation whose pole lies outside $\Gamma$.

Proof. It is easy to check that such a fractional linear transformation works. Conversely, suppose $h \circ \phi$ is a quotient of measures. If $\alpha<\gamma<\beta$ in $\Gamma$ choose a test set configuration as in Lemma 3.5. It follows from Lemma 3.4 that $\phi\left(A_{1} \vee B\right)$ is the harmonic conjugate of $\alpha$ with respect to the pair $(\beta, \gamma)$. But Lemma 3.4 also implies that the test set configurations of $h \circ \phi$ yield harmonic quadruples. Therefore $h$ preserves harmonic quadruples. Hence it is the restriction to $\Gamma$ of a fractional linear transformation.

This uniqueness theorem has an amusing statistical mechanical interpretation. Select a state of a given statistical mechanical system, that is, choose an atom free probability measure $\mu$ on the phase space $\mathfrak{M}$ of the system. Also choose an observable $f \in L^{1}(\mu)$. Perform experiments to compute the average value of $f$ on each subset of $\mathfrak{M}$ when the system is in state $\mu$. The average value will be undefined on $\mu$ null sets. Suppose you have lost all but the qualitative data, the partial ordering induced by the average value on the set of subsets of $\mathfrak{M}$ with positive $\mu$ measure. Then both the state $\mu$ and the observable $f$ can be recovered from this ordering, modulo the confusion of observable with state introduced by the arbitrary fractional linear transformation in Theorem 3.6. The larger the essential bounds of $f$ the less the confusion. If you happen to have chosen an $f$ unbounded above and below then $\mu$ is completely determined and $f$ is 
between $\phi(D)$ and $\phi(G)$ we can conclude that $\phi(G) \geqq \gamma>\phi(D \vee G) \geqq \phi(D)$. Therefore $\phi\left\lceil E \Im^{\prime}<\gamma\right.$.

\subsection{Lemma. $\mathfrak{U}_{\gamma}$ is a principal ideal. Call its generator $E_{\gamma}$.}

Proof. If $\gamma$ is below all of $\Gamma_{0}$ in $\Gamma$ set $E_{\gamma}=0$. If $\gamma$ is not below $\Gamma_{0}$ then $\mathfrak{A}_{\gamma}$ is not empty. Let $E_{\gamma}=\bigvee \mathfrak{U}_{\gamma}$; it clearly suffices to show $E_{\gamma} \in \mathfrak{U}_{\gamma}$. We shall do this by showing that the join $E$ of a maximal chain $\mathfrak{C}$ in $\mathfrak{A}_{\gamma}$ must equal $E_{\gamma}$ and lie in $\mathfrak{A}_{\gamma}$. $E$ lies in $\mathfrak{A}_{\gamma}$ because $\phi$ is continuous and $D \leqq E$ implies $D=\vee_{C \in \mathscr{G}} D \wedge C$. But if $F \in \mathfrak{A}_{\gamma}$ Lemma 4.2 implies $E \vee F \in \mathfrak{A}_{\gamma}$ so $E \vee F \leqq E$. Thus $E=E_{\gamma}$.

4.5. It is clear that the map $\gamma \rightarrow E_{\gamma}$ constructed in Theorem 4.4 is a spectral family. We shall call it the Radon-Nikodym derivative $\mathbb{E}$ of $\phi$. Note that $\alpha<\phi \uparrow\left(E_{\beta}-E_{\alpha}\right) \subseteq \leqq \beta$. To see this suppose $D \leqq E_{\beta}-E_{\alpha}$. Clearly $\phi(D) \leqq \beta$. But $D \perp E_{\alpha}$, so $\phi(D)>\alpha$ lest 4.3 applied to $\phi \uparrow D S$ contradict the maximality of $E_{\alpha}$.

4.6. THEOREM. Suppose $v$ is a finite signed measure absolutely continuous with respect to the finite measure $\mu$ on $(X, S)$. Then there is a function $f \in L^{1}(X, \mu)$ such that for all $A \in S, v(A)=\int_{A} f d \mu$.

Proof. $\phi=v / \mu$ is an averaging function on the measure algebra $\subseteq$ of $\mu$. The range $\Gamma_{0}$ of $\phi$ is included in $R$ so Theorem 4.4 and the remarks in 4.5 apply. Let $f$ be the measurable function defined by the Radon-Nikodym derivative of $\phi$.

Suppose $A \in S$. For integers $n>0$ and $m$ set $A_{m, n}=A \wedge\left(E_{(m+1) / 2^{n}}-E_{m / 2^{n}}\right)$. Then for each $n$, the join $A=\bigvee_{m} A_{m, n}$ is disjoint. Let $f_{n}$ be the function defined on $A$ by

$$
f_{n}(x)=v\left(A_{m, n}\right) \text { when } x \in A_{m, n}
$$

Then $f_{n}$ is well defined modulo $\mu$. Let us compute its integral with respect to $\mu$ over $A$. The following equalities are all true if any member converges.

$$
\begin{aligned}
\int_{A} f_{n} d \mu & =\sum_{m=-\infty}^{\infty} \phi\left(A_{m, n}\right) \mu\left(A_{m, n}\right) \\
& =\sum_{m} v\left(A_{m, n}\right) \\
& =v\left(\bigvee_{m} A_{m, n}\right) \\
& =v(A) .
\end{aligned}
$$

Thus for all $n, f_{n} \in L^{1}(A, \mu)$ and $\int_{A} f_{n} d \mu=v(A)$, independent of $n$. The definition of $f$ shows $\left|f_{n}-f\right|$ is almost everywhere less than $2^{-n}$. It follows that $f_{n} \rightarrow f$ uni- 
formly almost everywhere, so $f \uparrow A$ is in $L^{1}(A, \mu)$ and $\int_{A} f d \mu=\lim \int_{A} f_{n} d \mu$ $=v(A)$.

We return now to our study of averaging functions. Again we assume that $\mathfrak{S}$ is atom free and that $\phi: \widetilde{\varsigma}^{\prime} \rightarrow \Gamma$ is onto. $\mathbb{E}$ is the Radon-Nikodym derivative of $\phi$.

4.7. The point spectrum $\Lambda$ of $\phi$ is the set of those $\gamma$ in $\Gamma$ at which $\mathbb{E}$ is discontinuous. That is, $\gamma \in \Lambda$ when $F_{\gamma}=E_{\gamma}-\bigvee_{\beta<\gamma} E_{\beta} \neq 0$. Clearly $\gamma \in \Lambda$ if and only if there is an $F \neq 0$ such that $\phi \uparrow F \Im=\gamma$. If we constuct the Radon-Nikodym derivative $\mathbb{E}^{\prime}$ of the averaging function $\phi: \mathbb{S}^{\prime} \rightarrow \Gamma^{\prime}$ where $\Gamma^{\prime}$ is $\Gamma$ with the reverse order then for all $\gamma, E_{\gamma} \vee E_{\gamma}^{\prime}=1$ and $E_{\gamma} \wedge E_{\gamma}^{\prime}=F_{\gamma}$. When $\gamma \in \Lambda$ we shall call $F_{\gamma}$ the $\gamma$-atom. It is not an atom of $\mathbb{S}$, which has none. If $\Gamma$ has a greatest or a least element each is in $\Lambda$.

4.8. THEOREM. If $\Im$ is a measure algebra then $\Gamma$ is homeomorphic to a real interval.

Proof. The Radon-Nikodym derivative $\mathfrak{E}$ is a chain in $\mathfrak{S}$. Embed it in a maximal chain $\mathfrak{C}$. The remark in 4.5 and an argument analogous to the proof of Lemma 3.2 imply that $\phi$ is a strictly increasing function from $\mathfrak{C}$, which is an arc (1.6) onto the part of $\Gamma$ below $\phi(1)$. A similar argument applied to $\mathbb{E}^{\prime}$ finishes the proof.

5. More general averaging functions. In this section we construct a large class of averaging functions and gain new insight into the uniqueness theorem.

5.1. Let $\subseteq$ be an atom free measure algebra and $V$ the vector space of finite signed measures on $\mathfrak{S}$. Let $\boldsymbol{C}$ be the cone of nonnegative measures and $\boldsymbol{V}^{\prime}$ the set of strictly signed measures. Suppose $\Gamma$ is a linearly ordered set. Denote its greatest and least elements (if any) by $\gamma^{*}$ and $\gamma_{*}$ respectively. Let $\Gamma_{0}=\Gamma-\left\{\gamma^{*}, \gamma_{*}\right\}$.

5.2. TheOREM. Suppose $F^{*}$ and $F_{*}$ are disjoint elements of $\mathfrak{S}$, each less than 1 , and $F^{*}\left(F_{*}\right)>0$ if $\gamma^{*}\left(\gamma_{*}\right)$ exists. Suppose further that $\pi: \Gamma_{0} \rightarrow V^{\prime}$ is a function such that for every $A \in \mathfrak{S}^{\prime}, \gamma \rightarrow \pi_{\gamma}(A)$ is nondecreasing and

$$
\begin{aligned}
& \pi_{\gamma}(A)<0 \text { for all } \gamma \text { if } A \leqq F^{*}, \\
& \pi_{\gamma}(A)>0 \text { for all } \gamma \text { if } A \leqq F_{*}, \\
& \pi_{\gamma}(A)=0 \text { for exactly one } \gamma \in \Gamma_{0} \text { otherwise. }
\end{aligned}
$$

Define $\phi=\phi_{\pi}: \Im^{\prime} \rightarrow \Gamma$ by

$$
\phi(A)=\left\{\begin{array}{l}
\gamma^{*} \text { if } A \leqq F^{*}, \\
\gamma_{*} \text { if } A \leqq F_{*}, \\
\text { the solution } \gamma \text { to } \pi_{\gamma}(A)=0 \text { otherwise. }
\end{array}\right.
$$

Then $\phi$ is a continuous averaging function. 
Proof. We shall check only a few sample cases in the argument showing $\phi$ averages. Suppose that $A \perp B$ and that each meets both $1-F^{*}$ and $1-F_{*}$. If $\phi(A)<\phi(B)$ then

$$
\begin{aligned}
\pi_{\phi(A)}(A \vee B) & =\pi_{\phi(A)}(A)+\pi_{\phi(A)}(B) \\
& =\pi_{\phi(A)}(B)<0
\end{aligned}
$$

since $\gamma \rightarrow \pi_{\gamma}(B)$ is nondecreasing and vanishes only at $\phi(B)>\phi(A)$. Similarly, $\pi_{\phi(B)}(A \vee B)>0$. Thus $\phi(A \vee B)$, the zero of $\gamma \rightarrow \pi_{\gamma}(A \vee B)$, lies strictly between $\phi(A)$ and $\phi(B)$.

If $A \leqq F_{*}$, so that $\phi(A)=\gamma_{*}<\phi(B)$, then $\pi_{\phi(B)}(A \vee B)=\pi_{\phi(B)}(A)>0$. Therefore the zero of $\gamma \rightarrow \pi_{\gamma}(A \vee B)$ occurs before $\phi(B)$ and $\gamma_{*}<\phi(A \vee B)<\phi(B)$.

To show $\phi$ is continuous suppose $\mathfrak{C}$ is a chain in $\mathfrak{S}, C_{n} \rightarrow A$ in $\mathfrak{C}$ and $\alpha<\phi(A)<\beta$. Then $\pi_{\alpha}(A)<0<\pi_{\beta}(A)$. Since $\pi_{\alpha}$ and $\pi_{\beta}$ are measures each is continuous on $\mathbb{C}$ so eventually $\pi_{\alpha}\left(C_{n}\right)<0<\pi_{\beta}\left(C_{n}\right)$. Therefore eventually $\alpha<\phi\left(C_{n}\right)<\beta$.

5.3. Reparametrization of the curve $\gamma \rightarrow \pi_{\gamma}$ does not change the isomorphism class of $\phi$, for if $h: \Gamma^{\prime} \rightarrow \Gamma$ is an order isomorphism then Theorem 5.2 applied to the data $F^{*}, F_{*}$ and $\pi \circ h$ yields the averaging function $h^{-1} \circ \phi_{\pi}$.

5.4. There is another useful way to change $\pi$ without altering $\phi_{\pi}$. If $f$ is a positive function on $\Gamma$ such that for every $A, \gamma \rightarrow f(\gamma) \pi_{y}(A)$ is still nondecreasing then $\phi_{\pi}=\phi_{f \pi^{\prime}}$. Conversely, if $\phi_{\pi}=\phi_{\pi^{\prime}}$ then 1.23 shows $\pi$ and $\pi^{\prime}$ are related by such an $f$. In $\$ 7$ we discuss the existence of such renormalizations in some special cases.

5.5. TheOREM. For each $A$ in $\subseteq$ the function $\gamma \rightarrow \pi_{\gamma}(A)$ is continuous.

Proof. Suppose $\gamma \rightarrow \pi_{\gamma}(A)$ is discontinous at $\delta$. Note that whenever we write $A$ as a disjoint union $A_{1} \vee \cdots \vee A_{n}$ at least one of the functions $\gamma \rightarrow \pi_{\gamma}\left(A_{i}\right)$ is discontinuous at $\delta$. Furthermore if $B, \perp A$ then $\gamma \rightarrow \pi_{\gamma}(A \vee B)$ is discontinuous at $\delta$. The concluding remarks in 1.18 and 3.1 then show that when we use either of these constructions to replace $A$ by a subelement $A^{\prime}$ we can assume $\gamma \rightarrow \pi_{\gamma}\left(A^{\prime}\right)$ is discontinuous at $\delta$.

Assume for definiteness that $\pi_{\delta}(A) \geqq 0$. Since $\pi_{\delta}$ is strictly signed there is an $N$ for which $\pi_{\delta}(N)<0$. Lemma 3.1 shows we may assume $A \perp N$. Apply 1.18 sufficiently often to $\pi_{\delta}$ on $A$ to replace $A$ by a subelement $A^{\prime}$ such that $0 \leqq \pi_{\delta}\left(A^{\prime}\right)$ $<-\pi_{\delta}\left(N^{\prime}\right)$. Now let $p=\sup _{\gamma<\delta} \pi_{\gamma}\left(A^{\prime}\right)$ and $q=\inf _{y>\delta} \pi_{\gamma}\left(A^{\prime}\right)$. Then $p<q$ and $p \leqq \pi_{\delta}\left(A^{\prime}\right) \leqq q$. Finally, use 1.17 for some $t$ near $\pi_{\delta}\left(A^{\prime}\right) /-\pi_{\delta}(N)$ to find an $N^{\prime}<N$ such that $p<-\pi_{\delta}\left(N^{\prime}\right)<q$ and $-\pi_{\delta}\left(N^{\prime}\right) \neq \pi_{\delta}\left(A^{\prime}\right)$. Then $\pi_{\gamma}\left(A^{\prime} \vee N^{\prime}\right)$ is sometimes positive and sometimes negative but never zero, a contradiction.

5.6. Lemma. The span in $V$ of the range of $\pi$ meets $C$.

Proof. If $\alpha<\beta$ in $\Gamma_{0}$ then $\pi_{\alpha}$ and $\pi_{\beta}$ satisfy the hypotheses of Theorem 1.20. 
5.7. Let us examine a little more carefully the behavior of $\pi$ near the (top) end of $\Gamma$. Set

$$
v^{*}(A)=\sup _{\gamma \in \Gamma_{0}} \pi_{\gamma}(A)=\lim _{\gamma \uparrow \gamma^{*}} \pi_{\gamma}(A) .
$$

Then $v^{*}$ is a finitely additive but not necessarily finite signed measure. The following theorem shows that if it is finite and countably additive it is nonnegative. In $\$ 7$ we shall see that these remarks are in a sense the best possible.

5.8. THEOREM. There is no $v \in V^{\prime}$ such that $v \geqq \pi_{\gamma}$ for all $\gamma \in \Gamma_{0}$.

Proof. Suppose $v$ is such a measure. Whenever $v(A)<0, \pi_{\gamma}(A)<0$ for all $\gamma$ so $A \leqq F^{*}(5.2)$. Fix some $N$ for which $v(N)<0$; then find a $B \perp F^{*}$ small enough so that $v(N \vee B)<0$. Then $N \vee B \leqq F^{*}$, a contradiction.

5.9. If $\phi=v / \mu$ then $\phi=\phi_{\pi}$ for the curve $\pi$ given by $\pi_{\gamma}=\gamma \mu-\nu \in V^{\prime}$ for all $\gamma \in \Gamma_{0}$. Here $F^{*}\left(F_{*}\right)$ is the $\gamma^{*}\left(\gamma_{*}\right)$-atom, if any (4.7). Let $V_{2}$ be the two dimensional span of $v$ and $\mu$ in $V . C \cap V_{2}$ is either a sector or a ray. The lines - - - in the figure illustrate each possibility. We can now suddenly see why projective transformations occur in Theorem 3.6. Suppose we have already proved that every averaging function is of the type constructed in Theorem 5.2. It follows that if $v / \mu$ is isomorphic to $v^{\prime} / \mu^{\prime}$ then $v, \mu$ and $v^{\prime}, \mu^{\prime}$ are just different bases for the same subspace of $\boldsymbol{V}$ and one of the situations in the figure occurs: the relation between the ranges $-\ldots-\ldots$ and.$--\cdots$ is by projection from the origin.

5.10. THEOREM. $\phi_{\pi}$ is isomorphic to a quotient of measures if and only if the range of $\pi$ is contained in a plane.

Proof. 5.3 and 5.4 show that the span of the range of $\pi$ depends only on the isomorphism class of $\phi_{\pi}$. We have just observed that $\pi$ is a plane curve when $\phi=v / \mu$. Conversely, suppose $\pi\left(\Gamma_{0}\right) \subseteq V_{2}$. Lemma 5.6 shows $V_{2}$ meets $C$. Lemma 5.8 shows $\pi\left(\Gamma_{0}\right)$ meets every line through the origin in $V_{2} \cap V^{\prime}$. We know $\pi\left(\Gamma_{0}\right)$ meets each such line at most once by virtue of the hypotheses of Theorem 5.2. Theorem 5.5 shows that all of $\pi\left(\Gamma_{0}\right)$ lies in one of the two components into which $C \cup(-C)$ separates $V^{\prime} \cap V_{2}$. Therefore one of the curves in the figure gives an accurate picture of $\pi\left(\Gamma_{0}\right)$. In either case we can replace $\pi$ by $f \pi$ where $f$ is chosen so that $f(\gamma) \pi_{\gamma}$ lies on the line $-\ldots-\phi_{\pi}$ is isomorphic to $\phi_{f \pi}(5.4)$ which is in turn isomorphic to a quotient of measures (5.9).

This answers the question "When is $\phi$ isomorphic to a quotient of measures?" but the answer is unpleasant. In the next section we shall find a simple condition which forces $\pi\left(\Gamma_{0}\right)$ to be a plane curve and thus come to a more satisfactory answer.

\section{Every averaging function is a curve of measures.}

6.1. Let $\phi$ be an averaging function on $\mathfrak{S}$. We shall use Theorem 2.7 to construct a function $\pi$ for which $\phi=\phi_{\pi}$. It will follow that $\varsigma$ is a measure algebra. Tempo- 


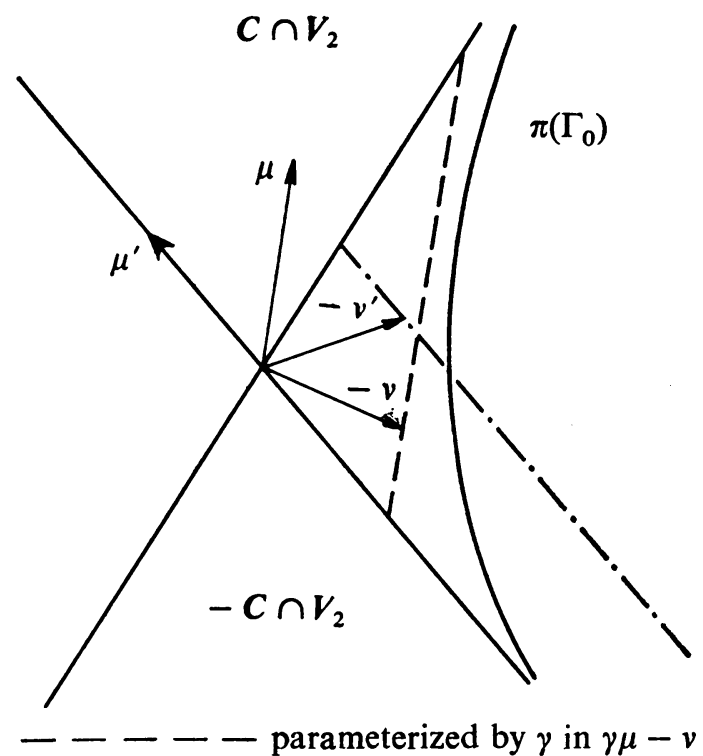

- - - - - parameterized by $\gamma$ in $\gamma \mu^{\prime}-v^{\prime}$

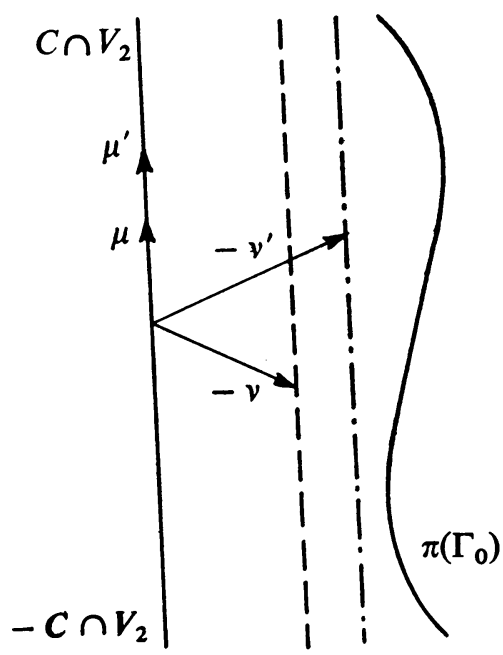

rarily fix $\gamma \in \Gamma_{0}$. Set $P_{0}=E_{\gamma}-F_{\gamma}(4.7), \mathfrak{P}_{\gamma}=P_{0} \subseteq, \mathfrak{R}_{\gamma}=\left(1-E_{\gamma}\right) \subseteq$ and $\mathfrak{S}_{\gamma}=\left(1-F_{\gamma}\right) \mathfrak{S}_{\text {. Recall that }} 3_{\gamma}=\phi^{-1}(\gamma)$. We wish to apply Theorem 2.7 to $3_{\gamma} \cap \mathcal{S}_{\gamma}$ and $P_{0}$ on $\mathfrak{S}_{\gamma}$. Hypothesis $I^{\prime}$ is clearly true.

6.2. Lemma. If $P \in \mathfrak{P}_{\gamma}$ and $N \in \mathfrak{N}_{\gamma}$ then $\phi(N \vee P) \geqq \gamma$ if and only if $P$ is balanced by some $N^{\prime}<N$. Dually, $N$ is balanced by a $P^{\prime}<P$ if and only if $\phi(N \vee P) \leqq \gamma$. 
Proof. Lemma 3.2 suffices to verify "only if" in the first assertion. Conversely, when such an $N^{\prime}$ exists $\phi(N \vee P) \geqq \gamma$ since it is between $\phi\left(N^{\prime} \vee P\right)=\gamma$ and

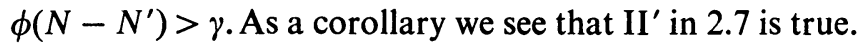

\subsection{LEMMA. $P_{0}$ and $\mathfrak{Z}_{\gamma} \cap \mathfrak{S}_{\gamma}$ satisfy $\mathrm{III}^{\prime}$.}

Proof. Suppose $N \in \mathfrak{N}_{\gamma}$ balances $P \in \mathfrak{P}_{\gamma}$ and $\mathfrak{C}$ is a maximal chain in $\mathfrak{P}_{\gamma}$. Since $E_{\gamma}-F_{\gamma}=P_{0} \geqq P$ and $\phi(N)>\gamma$ Lemma 6.2 implies $\phi\left(N \vee P_{0}\right) \leqq \gamma$. Then Lemma 3.2 applied to $P_{0}, N$ and $\mathfrak{C}$ produces a $C \in \mathbb{C}$ which balances $N$.

6.4. Let $\pi_{\gamma}$ be the strictly signed measure on $\mathfrak{S}_{\gamma}$ constructed in Theorem 2.7 from the data $P_{0}$ and $3_{\gamma} \cap \Im_{\gamma}$. Extend $\pi_{\gamma}$ to all of $\subseteq$ by setting $\pi_{\gamma}(A)=0$ whenever $A \leqq F_{\gamma}$.

6.5. THEOREM. $\mathfrak{S}$ is a measure algebra and $\Gamma$ is homeomorphic to a real interval.

Proof. The second assertion follows from the first (Theorem 4.8). We showed in 6.4 that $\left(1-F_{\phi(1)}\right) \subseteq$ is a measure algebra. If $\phi(1) \notin \Lambda$ we are done. In any case, if $\gamma_{*}<\gamma<\phi(1)$ then $F_{\phi(1)} \mathfrak{S} \subset \mathfrak{S}_{\gamma} . F_{\phi(1)} \mathfrak{S}$ and hence $\mathfrak{S}$ itself is therefore a measure algebra.

6.6. LEMMA. $\pi_{\gamma}(A)>0(<0)$ if and only if $\phi(A)<\gamma(>\gamma)$.

Proof. Write $A$ as a disjoint union $N \vee Z \vee P$ where $N \in \mathfrak{N}_{\gamma}, Z \leqq F_{\gamma}$ and $P \in \mathfrak{P}_{\gamma}$. Then $\pi_{\gamma}(A)=\pi_{\gamma}(N)+\pi_{\gamma}(P)>0$ if and only if $\pi_{\gamma}(P)>-\pi_{\gamma}(N)$. The definition of $\pi_{\gamma}$ together with Lemma 6.2 show this happens just when $\phi(N \vee P)<\gamma$. Since $\phi(Z)=\gamma, \phi(N \vee P)<\gamma$ if and only if $\phi(N \vee Z \vee P)<\gamma$

Next we fit the measures $\pi_{\gamma}$ together.

6.7. LEMMA. There is a positive real valued function $f$ on $\Gamma_{0}$ such that for every $A \in \mathbb{S}$ the function $\gamma \rightarrow f(\gamma) \pi_{\gamma}(A)$ is nondecreasing.

Proof. We shall arrange the construction so that $f(\phi(1))=1$. Note first that whenever $\gamma<\delta$ in $\Gamma_{0}$ Theorem 1.20 applies to $\pi_{\gamma}$ and $\pi_{\delta}$. When $\phi(1) \leqq \gamma$ set $I_{\gamma}=\left\{x: \pi_{\phi(1)} \leqq x \pi_{\gamma}\right\}$. When $\phi(1) \geqq \gamma$ set $I_{\gamma}=\left\{x: x \pi_{\gamma} \leqq \pi_{\phi(1)}\right\}$. Each $I_{\gamma}$ is a closed interval so their product $X$ is compact. For each finite subset $\Delta=\left\{\gamma_{1}, \cdots, \gamma_{n}\right\}$ of $\Gamma_{0}$ which contains $\phi(1)$ let $K(\Delta)$ be the subset of $X$ containing those functions $f$ for which

$$
f\left(\gamma_{1}\right) \pi_{\gamma_{1}} \leqq \cdots \leqq \pi_{\phi(1)} \leqq \cdots \leqq f\left(\gamma_{n}\right) \pi_{\gamma_{n}} .
$$

When $\Delta$ has two elements $K(\Delta)=X$. Induction on the cardinality of $\Delta$ shows $K(\Delta)$ is never empty. Any $f$ in $\bigcap K(\Delta)$ satisfies the conclusion of the theorem.

Henceforth we shall assume $\pi$ has been replaced by $f \pi$. We have proved

6.8. THEOREM. Suppose $\phi: \mathfrak{S}^{\prime} \rightarrow \Gamma$ is an averaging function on a complete atom free Boolean algebra $\mathfrak{S}$. Then $\mathfrak{S}$ is a measure algebra. Let $F^{*}\left(F_{*}\right)$ be the 
$\gamma^{*}\left(\gamma_{*}\right)$-atom of $\phi$ (if any) and $\pi$ the function from $\Gamma_{0}$ to $V^{\prime}$ constructed above. Then $F^{*}, F_{*}$ and $\pi$ satisfy the hypotheses of Theorem 5.2 and $\phi=\phi_{\pi}$.

6.9. We can now couple Theorems 5.10 and 6.8 to decide when $\phi=v / \mu$. Both the condition and the proof which follow are motivated by this observation: if $\phi=v / \mu$ then on each $3_{\gamma}, v$ is a constant multiple of $\mu$. Hence when restricted to $3_{\gamma}$ all the measures $\pi_{\delta}=\delta \mu-v$ lie on a line.

6.10. We shall call the averaging function $\phi$ impartial if

$$
\begin{gathered}
A_{1}, A_{2}, \text { and } B \text { mutually disjoint, } \\
\qquad \phi\left(A_{1}\right)=\phi\left(A_{2}\right) \neq \phi(B)
\end{gathered}
$$

and

imply

$$
\phi\left(A_{1} \vee B\right)=\phi\left(A_{2} \vee B\right)
$$

$$
\phi\left(A_{1} \vee C\right)=\phi\left(A_{2} \vee C\right) \text { for any } C \perp A_{1} \vee A_{2}
$$

Note that this last equality is trivially true whenever $\phi(C)=\phi\left(A_{1}\right) . \phi$ is impartial if whenever $\left\langle A_{1}, A_{2}, B\right\rangle$ forms a test set configuration (3.3), $C \perp A_{1} \vee A_{2}$ and $\phi(C) \neq \phi\left(A_{1}\right)$ it follows that $\left\langle A_{1}, A_{2}, C\right\rangle$ is also a test set configuration. Lemma 3.4 shows that every $\phi$ isomorphic to a quotient of measures is impartial.

6.11. Lemma. If $\gamma<\beta<\alpha$ in $\Gamma_{0}$ and $\phi$ is impartial then $\pi_{\beta}$ is a positive multiple of $\pi_{\alpha}$ on $3_{\gamma}$.

Proof. Since both $\pi_{\alpha}$ and $\pi_{\beta}$ are positive measures on $3_{\gamma}$ Lemma 2.6 shows that it suffices to prove they determine the same equivalence relation on $3_{\gamma}$. We shall show only that $\pi_{\beta}(E)=\pi_{\beta}(F)$ implies $\pi_{\alpha}(E)=\pi_{\alpha}(F)$ when $E, F \in \mathcal{Z}_{\gamma}$; the argument is almost symmetrical in $\alpha$ and $\beta$. Choose some $G$ in $\mathfrak{S}$ such that $\alpha<\phi(G)=\delta$. Apply Lemma 3.1 to the vector valued measure $v=\left\langle\pi_{\gamma}, \pi_{\beta}, \pi_{\alpha}, \pi_{\delta}\right\rangle$ and the elements $E, F$ and $G$ to find pairwise disjoint elements $E^{\prime}, F^{\prime}$ and $G^{\prime}$ such that $v\left(Y^{\prime}\right)=v(Y) / 8, Y=E, F, G$. Then in particular $\pi_{\gamma}\left(E^{\prime}\right)=\pi_{\gamma}\left(F^{\prime}\right)=0$ so $E^{\prime}$ and $F^{\prime}$ are still in $3_{\gamma}$. It clearly suffices to show $\pi_{\alpha}\left(E^{\prime}\right)=\pi_{\alpha}\left(F^{\prime}\right)$ so we may drop the primes and assume $E, F$ and $G$ disjoint from the start.

Now apply Corollary 1.18 sufficiently often to $v$ and $E, F$ to find subelements $E^{\prime}, F^{\prime}$ in $3_{\gamma}$ such that $\phi\left(E^{\prime} \vee G\right)>\alpha$ and $\phi\left(F^{\prime} \vee G\right)>\alpha$. This is possible since $\phi$ is continuous. Again it suffices to prove $\pi_{\alpha}\left(E^{\prime}\right)=\pi_{\alpha}\left(F^{\prime}\right)$ so drop the primes. We now know

$$
\phi(E \vee G)>\alpha>\beta>\gamma=\phi(E)
$$

We may therefore use Lemma 3.2 twice for $E$ and $G$ to find subelements $H_{\alpha}$ and $H_{\beta}$ of $G$ for which $\phi\left(E \vee H_{\alpha}\right)=\alpha$ and $\phi\left(E \vee H_{\beta}\right)=\beta$. Since $\pi_{\beta}(F)=\pi_{\beta}(E)$, $\pi_{\beta}\left(F \vee H_{\beta}\right)=\pi_{\beta}\left(E \vee H_{\beta}\right)=0$. Therefore $\phi\left(F \vee H_{\beta}\right)=\beta=\phi\left(E \vee H_{\beta}\right)$. But we 
also know $\phi(E)=\phi(F)=\gamma \neq \beta$ so the impartiality of $\phi$ implies $\phi\left(F \vee H_{\alpha}\right)$ $=\phi\left(E \vee H_{\alpha}\right)=\alpha$. Therefore $0=\pi_{\alpha}\left(F \vee H_{\alpha}\right)=\pi_{\alpha}\left(E \vee H_{\alpha}\right)$ and thus $\pi_{\alpha}(F)=\pi_{\alpha}(E)$.

6.12. THEOREM. An averaging function on a complete, atom free Boolean algebra is isomorphic to a quotient of measures if and only if it is impartial.

Proof. We have already observed that impartiality is necessary if $\phi$ is to be isomorphic to $v / \mu$. To prove it suffices we plan to use Theorem 5.10. We shall show that if $\gamma<\beta<\alpha$ in $\Gamma_{0}$ then the measures $\pi_{\gamma}, \pi_{\beta}$ and $\pi_{\alpha}$ are dependent. Let $v$ be the vector measure $\left\langle\pi_{\gamma}, \pi_{\beta}, \pi_{\alpha}\right\rangle$. Its range $X$ is convex in $R^{3}$ and contains 0 . Suppose it has an empty interior. Then it is contained in a plane $c x+b y+a z=0$ and the desired dependency $c \pi_{\gamma}+b \pi_{\beta}+a \pi_{\alpha}=0$ follows.

Thus it suffices to show the interior of $X$ is empty. Since $\pi_{\gamma}$ is strictly signed $X$ meets both the open half spaces defined by the plane $P_{x=0}$. Thus it suffices to show the interior of $X \cap P$ in $P$ is empty (1.21). But $X \cap P$ is the range of $v \uparrow 3_{\gamma}$. Lemma 6.11 shows this range is a line segment.

\section{Some examples.}

7.1. Let $I$ be the closed unit interval, $I_{0}$ its interior, $\mu$ Lebesgue measure on $I$ and $\mathfrak{S}$ the measure algebra of $\mu$. Suppose $g: I_{0} \times I_{0} \rightarrow R$ and $y \rightarrow g(x, y)$ increases for each fixed $x$. Define the measure $\pi_{y}=\pi_{y}^{g}$ on $\mathfrak{S}$ by

$$
\pi_{y}(A)=\int_{A} g(x, y) d \mu(x) .
$$

Set

$$
F^{*}=\{x: g(x, y)<0 \text { for all } y\}
$$

and

$$
F_{*}=\{x: g(x, y)>0 \text { for all } y\} \text {. }
$$

Then $F^{*}, F_{*}$ and $y \rightarrow \pi_{y}$ will often satisfy the hypotheses of Theorem 5.2.

7.2. For example, that will be so when $g$ is the restriction to $I_{0} \times I_{0}$ of a continuous function on $I \times I$ such that for all $x, g(x, 0)<0<g(x, 1)$. Unfortunately this continuity requirement is too strong for the examples we wish to study.

7.3. We shall exhibit a $g$ for which $y \rightarrow \pi_{y}$ has no nonconstant renormalizations in the sense of 5.4. Define $g$ so that

$$
\begin{gathered}
g(x, x)=0 \text { when } 0<x<1, \\
I g(x, 2 x)=1 \text { when } 0<x<1 / 2,
\end{gathered}
$$

Above the line $y=2 x$ and below the line $y=x, y \rightarrow g(x, y)$ is linear with slope $x$, $g(1-x, 1-y)=-g(x, y)$, all $x$ and $y$.

Elsewhere $g$ is arbitrary subject to the restriction that it be continuous and, for each $x$, an increasing function of $y$. 
One can then use the fact that $g(x, \cdot)$ is flat for small $x$ to show that if $f: I_{0} \rightarrow \boldsymbol{R}^{+}$ decreases between $y$ and $z$ then for $x$ sufficiently small

$$
f(z) \pi_{z}^{g}((0, x))<f(y) \pi_{y}^{g}((0, x)) .
$$

If $y<z$ and $f(y)<f(z)$ a similar inequality is valid below the main diagonal when $x$ is near 1 . Thus $y \rightarrow \pi_{y}$ can be renormalized only by constant functions $f$.

7.4. With this function $g$ as a tool we can build a $k$ for which $y \rightarrow \pi_{y}^{k}(A)$ does not strictly increase for every $A$. Suppose $h$ satisfies the requirements outlined in 7.2 and that $h$ is constantly 1 on an open patch above $y=x$ and below $y=1-x$. Then we can find an $A \in \mathbb{S}$ and $y<z \in I_{0}$ such that $\pi_{y}^{h}(A)=\pi_{z}^{h}(A)=\mu(A)$. However, $y \rightarrow \pi_{y}^{h}$ may be renormalizable so as to correct this. To guard against the possibility, define $k$ by

$$
k(x, y)= \begin{cases}g(2 x, y), & 0<x \leqq 1 / 2 \\ h(2 x-1, y), & 1 / 2<x<1\end{cases}
$$

Then $y \rightarrow \pi_{y}^{k}$ is not renormalizable and does what we ask of it.

7.5. To illustrate the remarks in 5.7 we shall add a restriction on $g$. We require that it be almost uniformly unbounded:

$$
g(x, 1-(1 / n))=n \text { when } \frac{2}{n}<x<1-\frac{2}{n}, \quad n>5 .
$$

Then $y \rightarrow \pi_{y}^{g}$ is still not renormalizable. The measure $v^{*}$ constructed in 5.7 is identically $+\infty$ on $\mathfrak{S}^{\prime}$.

7.6. If we append a $\gamma^{*}$-atom by defining

$$
j(x, y)= \begin{cases}g(2 x, y), & 0<x \leqq 1 / 2, \\ -1, & 1 / 2<x<1\end{cases}
$$

then $F^{*}=(1 / 2,1)$ and $v^{*}$ is given by

$$
v^{*}(A)= \begin{cases}+\infty, & \text { if } \mu(A \cap(0,1 / 2))>0, \\ -\mu(A \cap(1 / 2,1)), & \text { otherwise. }\end{cases}
$$

$v^{*}$ is countably additive and strictly signed but not even $\sigma$-finite. Since no renormalization of $y \rightarrow \pi_{y}^{j}$ is possible this pathology is uncorrectable.

\section{REFERENCES}

1. L. E. Dubins and E. H. Spanier, How to cut a cake fairly, Amer. Math. Monthly 68 (1961), 1-17.

2. P. R. Halmos, The range of a vector measure, Bull. Amer. Math. Soc. 54 (1948), 416-421.

3. A. Liapounoff, Sur les fonctions-vecteurs completement additives, Bull. Acad. Sci. URSS Sér. Math. 4 (1940), 465-478. 
4. D. Maharam, The representation of abstract measure functions, Trans. Amer. Math. Soc. 65 (1949), 279-330.

5. J. von Neumann, Continuous geometry, Princeton Mathematical Series No. 25, Princeton Univ. Press, Princeton, N. J., 1960.

6. P. D. Finch, A generalization of the Radon-Nikodym Theorem, J. Austral. Math. Soc. 5 (1965), 17-24.

Bryn MaWr College,

Bryn Mawr, Pennsylvania 